\title{
Toward a Network of Connected Things
}

DEEPAK VASISHT, CSAIL, MIT

Research for Practice combines the resources of the ACM Digital Library, the largest collection of computer science research in the world, with the expertise of the ACM membership. In every

RfP column experts share a short curated selection of papers on a concentrated, practically oriented topic.

(4) his installment of Research for Practice features a curated selection from Deepak Vasisht, who takes us on a tour of systems and networking for the Internet of Things. Deepak's selection spans energy harvesting to agriculture, providing a look into the future of loT deployments and their usability.

-Peter Bailis

Over the past few years, we have started realizing the loT (Internet of things) dream. Amazon Echo, Dash buttons, Nest cameras, Google Home, etc. have permeated our lives at home, and enterprises in various sectors such as retail, airlines, transportation and logistics have started benefiting from industrial loT solutions. Inspired by this impetus, General Electric recently estimated that investments in industrial loT alone would top $\$ 60$ trillion over the next 15 years.

All this growth has been fueled by years of research tackling several challenges, ranging from low-power networking to new sensor designs to security and privacy. This installment of Research for Practice presents research papers that aim: (a) to make loT deployments 
more pervasive and [b] to enable users to gain more utility from existing deployments.

\section{Easing the cost of deployment}

Zhang, P., Bharadia, D., Joshi, K., Katti, S. 2016. HitchHike: practical backscatter using commodity Wi-Fi. Proceedings of the 14th ACM Conference on Embedded Network Sensor Systems CD-ROM (SenSys):259-271; https:Ildl.acm.orgl citation.cfm?id=2994565.

ne of the natural challenges of large-scale networked sensor deployments is the cost of powering them up. The high power cost of communication modules leads to frequent battery replacements, which, in turn, incur large labor costs. A recent sequence of backscatter solutions aims to change that by leveraging existing radio frequency transmissions to communicate. Specifically, backscatter communication systems allow devices to modulate and reflect existing Wi-Fi transmissions, thus enabling lowpower communication modules that could be powered either by harvesting ambient power or by batteries that last several years.

HitchHike is unique for two reasons. First, not only can it reflect transmissions from commodity Wi-Fi devices, its reflections can also be received and decoded by commodity Wi-Fi devices. This allows the widely prevalent Wi-Fi devices, such as your access point, to interact with the sensors at a very low cost for power. Second, HitchHike can achieve a data rate of $200 \mathrm{Kbps}$ at a distance of 54 meters. This data rate is high enough for most sensors 
and covers a very large area, larger than most homes and small enterprises. Going forward, HitchHike and others in this space promise to allow applications with severe power constraints, such as implantable sensors, wearables, sensors embedded in walls and bridges, etc.

\section{Designing novel sensing mechanisms}

Adib, F., Mao, H., Kabelac, Z., Katabi, D., Miller, R. C. 2015.

Smart homes that monitor breathing and heart rate. Proceedings of the 33rd Annual ACM Conference on Human Factors in Computing Systems: 837-846; https:I/dl.acm.orgl citation.cfm?id=2702200.

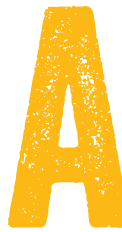

continuous thread of innovation in the loT space has been the design of novel sensing mechanisms. A recent trend in this space has been monitoring health metrics such as breathing, heart rate, walking patterns, sleep stages, gait, and even emotional health in a completely contactless way. Amazon Echo or Google Home, for example, could be equipped with these capabilities, allowing users to know more about their physical and mental health.

This paper describes VitalRadio, a device that can monitor breathing and heart rate of a user without any contact with the user at distances up to eight meters, even when the user is in a different room. VitalRadio presents the basic techniques that form the foundation of a lot of the later work on monitoring various other health metrics. On a high level, VitalRadio works by analyzing the reflections of radio signals from human bodies. As humans breathe (or their hearts beat], the reflections are affected by any 
minute change. VitalRadio extracts these small changes in the reflections to estimate the heart rate and breathing of individuals. While there are still some limitations on the operation of the system, like requirements for a one- to two-meter minimum separation between multiple users and quasi-static user behavior (watching TV, typing, etc.], none of these limitations is big enough to hinder mainstream health monitoring applications.

\section{Exploiting already-deployed sensors for new services} Abari, O., Vasisht, D., Katabi, D., Chandrakasan, A. 2015. Caraoke: an e-toll transponder network for smart cities. Proceedings of the 2015 ACM Conference on Special Interest Group on Data Communication: 297-310; https:II dl.acm.org/citation.cfm?id=2787504.

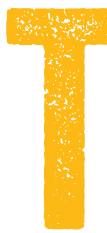
he push for loT over the past decade has ensured that we have already deployed several billion sensors, such as toll transponders in cars, RFIDs in warehouses and restaurants, etc. These devices enable very specific functionality-automatic toll collection for cars or inventory tracking in warehouses. A key question, then, is what can be done to leverage these large deployments for more general-purpose applications?

Caraoke achieves this for e-toll transponders by using them to monitor traffic, locate and identify cars, detect speeding, and enable automated detection of empty parking spots-all without any changes to the e-toll transponders deployed on cars. Since such transponders are being used by 70-89 percent of drivers in the U.S. (depending on the state) and are seeing increased adoption worldwide, Caraoke can 
play an important role in the push for smart cities where the traffic lights react to real-time traffic information, and drivers are automatically guided to empty parking spots. The fundamental contribution of Caraoke is its ability to separate simultaneous transmissions from multiple transponders using novel signal processing techniques that exploit the frequency-domain structure of the signal. Caraoke incorporates these techniques into a new reader for transponders that can be deployed on street-light poles and harvest solar energy for their operation. Going forward, such innovations in different domains can expand the utility of deployed loT systems manifold.

\section{Expanding loT to "untouched" environments}

Vasisht, D., Kapetanovic, Z., Won, J., Jin, X., Chandra, R., Kapoor, A., Sinha, S. N., Sudarshan, M., Stratman, S. 2017. FarmBeats: an loT platform for data-driven agriculture. Proceedings of the $14^{\text {th }}$ Usenix Symposium on Networked Systems Design and Implementation; https:/lwww.usenix.orgl conferencelnsdi17/technical-sessions/presentation/vasisht.

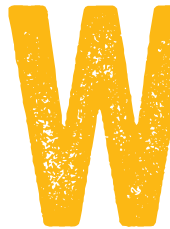

hile loT has prospered in well-connected, wellpowered environments such as urban homes and large enterprises, its adoption in harsher environments, without good sources of power and Internet, has been relatively low. Such environments include farming, construction, and miningsectors that employ large sections of both the developing and the developed world. For example, even in the United States, the process of data collection in farming remains primarily manual, which limits the adoption of advanced 
agricultural techniques to fewer than 20 percent of farmers.

FarmBeats attempts to tackle this challenge by focusing on the challenge of data-driven agriculture. Datadriven agricultural techniques such as precision irrigation can allow farmers to improve yields, reduce input cost, and enhance labor productivity. FarmBeats lets farmers employ these techniques by developing an end-to-end loT platform for agriculture that enables seamless data collection from sensors, cameras, and drones.

FarmBeats uses three ideas to enable this platform. First, to enable connectivity on the farm, it uses a mix of TV white spaces (to allow long-range connectivity over several miles) and Wi-Fi (to allow interfacing with commercial sensors). Second, to deal with weatherrelated outages and low bandwidths, it designs an loT gateway that sits on the farm and provides services to the farmer while creating summaries for the cloud. Finally, it leverages machine-learning techniques to combine inputs from a drone and ground sensors to provide more accurate information and reduce the requirement for sensor deployments. The paper presents results from a multiseason deployment of FarmBeats on two different farms on the two coasts of the United States.

\section{Final thoughts}

The exciting research being done in loT systems has put us ever closer not only to developing new services, but also to gathering new data sets for consumer and enterprise applications. Combined with recent significant advances in artificial intelligence and machine learning, these data sets can drive new applications. For example, VitalRadio has 
been extended to leverage novel deep-learning techniques to monitor sleep stages of a user completely passively. Researchers can also leverage new machine learning techniques as tools to design better systems. FarmBeats already shows how one can leverage Al to reduce the requirement for sensor placement and to guide the placement of sensors to maximize information.

While this scale of data presents new avenues for improvement, the key challenges for the everyday adoption of loT systems revolve around managing this data. First, we need to consider where the data is being processed and stored-on the local edge computer or in the cloud-and what the privacy and systems implications of these policies are. Second, we need to develop systems that generate actionable insights from this diverse, hard-to-interpret data for non-tech users. Solving these challenges will allow loT systems to deliver maximum value to end users.

Deepak Vasisht is a PhD candidate in electrical engineering and computer science at MIT. His research is focused on wireless networks and loT systems. He has designed, built and deployed systems that deliver ubiquitous sensing, accurate indoor positioning, enhanced communication capabilities, and new human computer interfaces. His research has been featured in the Economist, IEEE Spectrum, BBC, and CBC, among others. He is the recipient of the ACM SIGCOMM Best Paper award, a Microsoft Research PhD Fellowship, and the President's Gold Medal at IIT Delhi.

Copyright (C) 2017 held by ownerlauthor. Publication rights licensed to ACM. 